Pacific

Journal of

Mathematics

SMOOTH APPROXIMATION OF CONIC KÄHLER METRIC WITH LOWER RICCI CURVATURE BOUND

LIANGMING SHEN 


\title{
SMOOTH APPROXIMATION OF CONIC KÄHLER METRIC WITH LOWER RICCI CURVATURE BOUND
}

\author{
LIANGMING SHEN
}

\begin{abstract}
We apply methods in a paper of Tian (Comm. Pure Appl. Math. 68:7 (2015), 1085-1156) to prove that a conic Kähler metric with lower Ricci curvature bound can be approximated by smooth Kähler metrics with the same lower Ricci curvature bound. Furthermore, conic singularities here can be along a simple normal crossing divisor.
\end{abstract}

\section{Introduction}

Recently, very important progress has been made on Kähler-Einstein metrics on Fano manifolds (see [Tian 2015; Chen et al. 2015a; 2015b; 2015c]). The main tool is an extension of Cheeger-Colding-Tian theory [Cheeger et al. 2002] to conic Kähler-Einstein metrics. This extension allows one to establish a partial $C^{0}$-estimate, which has long been known to be crucial in proving the existence of Kähler-Einstein metrics. To extend Cheeger-Colding-Tian theory from the smooth case to the conic case, Tian [2015] proved a sharp approximation theorem: any conic Kähler-Einstein metric can be approximated by smooth Kähler metrics with the same lower Ricci curvature bound in the Cheeger-Gromov sense.

The main idea for proving this sharp approximation came from [Tian 2000], which gives a method of proving the equivalence of the $C^{0}$-estimate and the properness of the Lagrangian of the corresponding complex Monge-Ampère equation. Let's describe this in more detail. First, we can define the so-called twisted Ding energy $F_{\omega}(\varphi)$ and the twisted Mabuchi energy $v_{\omega}(\varphi)$ as in [Li and Sun 2014]; they are Lagrangians of the corresponding complex Monge-Ampère equation for the conic Kähler-Einstein metric. Then we can prove these two energies are both proper with respect to the functional $J_{\omega}(\varphi)$. After that, we perturb this singular complex Monge-Ampère equation, and prove that the corresponding energies are also proper after such a perturbation. Then, we make use of the $C^{0}$-estimate in [Tian 2012] to get a new $C^{0}$-estimate for the perturbed complex Monge-Ampère equation. Finally, according to the compactness theorem, we can prove that the perturbed

MSC2010: 53C55.

Keywords: conic metrics, Ricci curvature. 
Kähler metrics converge to the original conic Kähler-Einstein metric in the CheegerGromov sense, and converge smoothly in the $C^{\infty}$ sense outside the divisor.

Now a more general problem is to understand the structures of Kähler manifolds with lower Ricci curvature bound. A natural question is whether we can also approximate an arbitrary conic Kähler metric by smooth Kähler metrics with the same lower Ricci curvature bound. We observe that the method in [Tian 2015] applies if we can get suitable complex Monge-Ampère equations and define suitable energies for them. Moreover, instead of multiple anticanonical divisors as in the original proof, we can generalize our result to simple normal crossing divisors. A divisor $D$ is called a simple normal crossing divisor if it can be written as

$$
D=\sum_{i=1}^{m} D_{i}
$$

where each $D_{i}$ is an irreducible divisor, and they cross only in a transversal way. Each point $p \in D$ lies in the intersection of $k$ divisors, say $D_{1}, \ldots, D_{k}$, and in the local coordinate neighborhood $U$ we can write $D_{i}=\left\{z_{i}=0\right\}$. Assume that our conic Kähler metric $\omega$ on the Kähler manifold $M$ takes an angle $2 \pi \beta_{i}$ along each $D_{i}$, where $0<\beta_{i}<1$. Then near the point $p \in D$ which lies in the intersection of all $D_{i}$, the metric $\omega$ is asymptotically equivalent to the model conic metric

$$
\omega_{0, p}=\sqrt{-1}\left(\sum_{i=1}^{k} \frac{d z_{i} \wedge d \bar{z}_{i}}{\left|z_{i}\right|^{2\left(1-\beta_{i}\right)}}+\sum_{i=k+1}^{n} d z_{i} \wedge d \bar{z}_{i}\right) .
$$

We say a smooth Kähler metric $\omega_{0}$ on $M$ has a lower Ricci curvature bound $\mu$ if there exists a nonnegative $(1,1)$-form $\Omega_{0}$ such that

$$
\operatorname{Ric} \omega_{0}=\mu \omega_{0}+\Omega_{0} .
$$

And we say our conic Kähler metric $\omega$ has a lower Ricci curvature bound $\mu$ if there exists a nonnegative $(1,1)$-form $\Omega$ such that

$$
\operatorname{Ric} \omega=\mu \omega+\sum_{i=1}^{k} 2 \pi\left(1-\beta_{i}\right)\left[D_{i}\right]+\Omega
$$

(we may assume that $\Omega \neq 0$; otherwise we come back to the conic Kähler-Einstein case). This equation is in the sense of currents on $M$ and in the classic sense outside the singular part $D$. Considering these equations and applying Tian's methods for conic Kähler-Einstein metrics, we can prove our main theorem.

Theorem 1.1. For a Kähler manifold $(M, D)$, where $D$ is a simple normal crossing divisor, assume that we have a smooth background Kähler metric $\omega_{0}$ and a conic Kähler metric $\omega=\omega_{0}+\sqrt{-1} \partial \bar{\partial} \varphi$ with cone angle $2 \pi \beta_{i}\left(0<\beta_{i}<1,1 \leq i \leq m\right)$ 
along each irreducible component $D_{i}$ of $D$ and that $\varphi$ is a smooth real function on $M \backslash D$. If the conic Kähler metric $\omega$ has a lower Ricci curvature bound $\mu$, or $\omega$ is a conic Kähler-Einstein metric with Ricci curvature constant $\mu$ and an extra condition that $M$ does not have holomorphic fields, then for any $\delta>0$, there exists a smooth Kähler metric $\omega_{\delta}$ with the same lower Ricci curvature bound $\mu$ which converges to $\omega$ in the Gromov-Hausdorff topology on $M$ and in the smooth topology outside $D$ as $\delta$ tends to 0 .

Note that here we can deal with all the cases for $\mu$. However, by work of Aubin and Yau, the cases $\mu<0$ and $\mu=0$ are easy to handle. The difficulty will be when $\mu>0$, i.e., the Fano case. In the following section, we set up the complex Monge-Ampère equation and perturb it, and derive a $C^{0}$-estimate for nonpositive $\mu$. We deal with the case $\mu>0$ in the remaining parts of this paper.

\section{Basic setup and the case $\mu \leq 0$}

First, comparing equations (1-1) and (1-2), we have

$$
\sqrt{-1} \partial \bar{\partial} \log \frac{\omega^{n}}{\omega_{0}^{n}}=-\mu \varphi+\Omega_{0}-\Omega-\sum_{i=1}^{m}\left(1-\beta_{i}\right)\left(R\left(\|\cdot\|_{i}\right)+\sqrt{-1} \partial \bar{\partial} \log \left\|S_{i}\right\|_{i}^{2}\right),
$$

where $\omega=\omega_{0}+\sqrt{-1} \partial \bar{\partial} \varphi$ is the conic Kähler metric. As each $D_{i}$ is an irreducible positive divisor, we set $S_{i}$ as its defining holomorphic section, with $\left(\|\cdot\|_{i}\right)$ as the Hermitian product on the associated line bundle $\left[D_{i}\right]$, and the curvature of this bundle is defined as $R\left(\|\cdot\|_{i}\right):=-\sqrt{-1} \partial \bar{\partial} \log \|\cdot\|_{i}^{2}$. Then we get the equation above just from the Poincaré-Lelong equation

$$
2 \pi[D]=\sqrt{-1} \partial \bar{\partial} \log |S|^{2}=\sqrt{-1} \partial \bar{\partial} \log \|S\|^{2}+R(\|\cdot\|) .
$$

Noting that the left-hand sides of (1-1) and (1-2) both lie in the cohomology class $c_{1}(M)$, we deduce that

$$
\Omega_{0}-\Omega-\sum_{i=1}^{m}\left(1-\beta_{i}\right) R\left(\|\cdot\|_{i}\right)=\sqrt{-1} \partial \bar{\partial} h_{0},
$$

where $h_{0}$ is a smooth function on $M$, and we note that $\frac{1}{2 \pi} R\left(\|\cdot\|_{i}\right)$ represents $c_{1}\left(D_{i}\right)$. Then we get our complex Monge-Ampère equation:

$$
\left(\omega_{0}+\sqrt{-1} \partial \bar{\partial} \varphi\right)^{n}=e^{h_{0}-\mu \varphi-\sum_{i=1}^{m}\left(1-\beta_{i}\right) \log \left\|S_{i}\right\|_{i}^{2}+c} \omega_{0}^{n},
$$

where the constant $c$ is chosen so that

$$
\int_{M}\left(e^{h_{0}-\sum_{i=1}^{m}\left(1-\beta_{i}\right) \log \left\|S_{i}\right\|_{i}^{2}+c}-1\right) \omega_{0}^{n}=0 .
$$


As in [Tian 2015], we can choose such an approximation equation:

$$
\left(\omega_{0}+\sqrt{-1} \partial \bar{\partial} \varphi\right)^{n}=e^{h_{\delta}-\mu \varphi} \omega_{0}^{n},
$$

where

$$
h_{\delta}=h_{0}-\sum_{i=1}^{m}\left(1-\beta_{i}\right) \log \left(\delta+\left\|S_{i}\right\|_{i}^{2}\right)+c_{\delta}
$$

and the constant $c_{\delta}$ is chosen such that

$$
\int_{M}\left(e^{h_{0}-\sum_{i=1}^{m}\left(1-\beta_{i}\right) \log \left(\delta+\left\|S_{i}\right\|_{i}^{2}\right)+c_{\delta}}-1\right) \omega_{0}^{n}=0 .
$$

Here $c_{\delta}$ is uniformly bounded. If we have a solution $\varphi_{\delta}$ for (2-3), then we get a smooth Kähler metric $\omega_{\delta}=\omega_{0}+\sqrt{-1} \partial \bar{\partial} \varphi_{\delta}$ with Ricci curvature given by

$$
\begin{aligned}
\operatorname{Ric} \omega_{\delta}= & \operatorname{Ric} \omega_{0}+\mu \sqrt{-1} \partial \bar{\partial} \varphi_{\delta}-\sqrt{-1} \partial \bar{\partial} h_{\delta} \\
= & \mu \omega_{0}+\Omega_{0}+\mu \sqrt{-1} \partial \bar{\partial} \varphi_{\delta}-\sqrt{-1} \partial \bar{\partial} h_{0}+\sum_{i=1}^{m}\left(1-\beta_{i}\right) \sqrt{-1} \partial \bar{\partial} \log \left(\delta+\left\|S_{i}\right\|_{i}^{2}\right) \\
= & l \mu \omega_{\delta}+\Omega \\
& \quad+\sum_{i=1}^{m}\left(1-\beta_{i}\right)\left(R\left(\|\cdot\|_{i}\right)+\frac{\left\|S_{i}\right\|_{i}^{2}}{\delta+\left\|S_{i}\right\|_{i}^{2}} \sqrt{-1} \partial \bar{\partial} \log \left\|S_{i}\right\|_{i}^{2}+\frac{\delta D S_{i} \wedge \overline{D S_{i}}}{\left(\delta+\left\|S_{i}\right\|_{i}^{2}\right)^{2}}\right) \\
= & \mu \omega_{\delta}+\Omega+\sum_{i=1}^{m}\left(1-\beta_{i}\right)\left(\frac{\delta}{\delta+\left\|S_{i}\right\|_{i}^{2}} R\left(\|\cdot\|_{i}\right)+\frac{\delta D S_{i} \wedge \overline{D S_{i}}}{\left(\delta+\left\|S_{i}\right\|_{i}^{2}\right)^{2}}\right) .
\end{aligned}
$$

Note that $\left\|S_{i}\right\|_{i}^{2} \sqrt{-1} \partial \bar{\partial} \log \left|S_{i}\right|_{i}^{2}=\left\|S_{i}\right\|_{i}^{2} \cdot 2 \pi\left[D_{i}\right]=0$. We can see that if we have a solution $\varphi_{\delta}$ for small $\delta>0$, the Ricci curvature of $\omega_{\delta}$ is always greater than $\mu$.

By the computation above, we have a corollary which asserts the openness of the solvable set for the continuity path below.

Lemma 2.1. Consider the continuity path of (2-3),

$$
\left(\omega_{0}+\sqrt{-1} \partial \bar{\partial} \varphi\right)^{n}=e^{h_{\delta}-t \varphi} \omega_{0}^{n},
$$

which corresponds to the equation

$$
\operatorname{Ric} \omega_{t}:=\operatorname{Ric}\left(\omega_{0}+\sqrt{-1} \partial \bar{\partial} \varphi\right)=t \omega_{t}+(\mu-t) \omega_{0}+\Omega-\sqrt{-1} \partial \bar{\partial} h_{\delta},
$$

and set the interval $I_{\delta}$ as its solvable interval. Then $0 \in I_{\delta}$ and this interval is open. Proof. That $0 \in I_{\delta}$ follows from the Calabi-Yau theorem. By the computation above and [Tian 2015], it's easy to see that $\lambda_{1}\left(-\Delta_{t}\right)$ is strictly larger than $t$. Then the openness of $I_{\delta}$ follows. 
So now, to solve (2-3), we need to set up a $C^{0}$-estimate for $\varphi_{\delta}$. We first consider the cases $\mu=0$ and $\mu<0$. Actually, by the Calabi-Yau theorem and Aubin's work (see [Yau 1978]), we can get $C^{0}$-estimates for these cases. The main difficulty lies in the case $\mu>0$, which we will deal with in the following sections.

\section{Twisted functionals for complex Monge-Ampère equations, bounded from below}

Following [Berman 2013; Ding and Tian 1992; Jeffres et al. 2016; Tian 2000; Li and Sun 2014], we can still define corresponding functionals for our complex Monge-Ampère equation (2-2). First, we define generalized energy functionals.

Definition 3.1. We have

(1) $J_{\omega_{0}}(\varphi)=\frac{1}{V} \sum_{i=0}^{n-1} \frac{i+1}{n+1} \int_{M} \sqrt{-1} \partial \varphi \wedge \bar{\partial} \varphi \wedge \omega_{0}^{i} \wedge \omega_{\varphi}^{n-i-1}$,

(2) $I_{\omega_{0}}(\varphi)=\frac{1}{V} \int_{M} \varphi\left(\omega_{0}^{n}-\omega_{\varphi}^{n}\right)$,

where $V=\int_{M} \omega_{0}^{n}$ and $\omega_{\varphi}=\omega_{0}+\sqrt{-1} \partial \bar{\partial} \varphi$.

Note that these functionals are well defined even in the conic case. It's easy to check that

$$
0 \leq \frac{n+1}{n} J_{\omega_{0}}(\varphi) \leq I_{\omega_{0}}(\varphi) \leq(n+1) J_{\omega_{0}}(\varphi) .
$$

Next let's define two functionals which are both Lagrangians of (2-2). For simplicity here we set

$$
H_{0}=h_{0}-\sum_{i=1}^{m}\left(1-\beta_{i}\right) \log \left\|S_{i}\right\|_{i}^{2}+c,
$$

and we can choose a family $\varphi_{t}$ connecting 0 and $\varphi$.

Definition 3.2. (1) We define the twisted Ding functional as

$$
F_{\omega_{0}, \mu}(\varphi)=J_{\omega_{0}}(\varphi)-\frac{1}{V} \int_{M} \varphi \omega_{0}^{n}-\frac{1}{\mu} \log \left(\frac{1}{V} \int_{M} e^{H_{0}-\mu \varphi} \omega_{0}^{n}\right) .
$$

(2) We define the twisted Mabuchi functional as

$$
\begin{aligned}
v_{\omega_{0}, \mu}(\varphi) & =-\frac{n}{V} \int_{0}^{1} \int_{M} \dot{\varphi}\left(\operatorname{Ric} \omega_{\varphi}-\mu \omega_{\varphi}-\sum_{i=1}^{m} 2 \pi\left(1-\beta_{i}\right)\left[D_{i}\right]-\Omega\right) \wedge \omega_{\varphi}^{n-1} d t \\
& =\frac{1}{V} \int_{M} \log \frac{\omega_{\varphi}^{n}}{\omega_{0}^{n}} \omega_{\varphi}^{n}+\frac{1}{V} \int_{M} H_{0}\left(\omega_{0}^{n}-\omega_{\varphi}^{n}\right)-\mu\left(I_{\omega_{0}}(\varphi)-J_{\omega_{0}}(\varphi)\right) \\
& =\frac{1}{V} \int_{M} \log \frac{\omega_{\varphi}^{n}}{\omega_{0}^{n}} \omega_{\varphi}^{n}+\frac{1}{V} \int_{M} H_{0}\left(\omega_{0}^{n}-\omega_{\varphi}^{n}\right)+\mu\left(F_{\omega_{0}}^{0}(\varphi)+\frac{1}{V} \int_{M} \varphi \omega_{\varphi}^{n}\right),
\end{aligned}
$$


where

$$
F_{\omega_{0}}^{0}(\varphi)=J_{\omega_{0}}(\varphi)-\frac{1}{V} \int_{M} \varphi \omega_{0}^{n}
$$

These definitions are similar to the smooth case [Tian 2012] and the conic KählerEinstein case [Li and Sun 2014]. We can check that they are well defined for the conic case. From those papers, we know that to get a $C^{0}$-estimate for $\varphi_{\delta}$, we need to prove the corresponding twisted Ding functional is proper with respect to the generalized energy $J_{\omega_{0}}(\varphi)$. Now let's recall the definition of properness.

Definition 3.3. Suppose the twisted Ding functional $F_{\omega, \mu}(\varphi)$ (twisted Mabuchi functional $\left.v_{\omega, \mu}(\varphi)\right)$ is bounded from below, i.e., $F_{\omega, \mu}(\varphi) \geq-c_{\omega}\left(v_{\omega, \mu}(\varphi) \geq-c_{\omega}\right)$. We say it is proper on $P_{c}(M, \omega)$ if there is an increasing function $f:\left[-c_{\omega}, \infty\right) \rightarrow \mathbb{R}$, and $\lim _{t \rightarrow \infty} f(t)=\infty$, such that for any $\varphi \in P_{c}(M, \omega)$,

$$
F_{\omega, \mu}(\varphi) \geq f\left(J_{\omega}(\varphi)\right) \quad\left(v_{\omega, \mu}(\varphi) \geq f\left(J_{\omega}(\varphi)\right)\right),
$$

where $\varphi \in P_{c}(M, \omega)$ is a bounded function which is smooth on $M \backslash D$, and such that $\omega_{\varphi}=\omega+\sqrt{-1} \partial \bar{\partial} \varphi$ is a conic metric with the prescribed angles along each component of $D$.

There are a lot of properties for these functionals, which are parallel to those in [Tian 2012; Li 2012; Li and Sun 2014]. We just put two basic facts here; the proofs are in [Tian 2012; Li and Sun 2014].

Proposition 3.4. (1) Given a path $\left\{\varphi_{t}\right\}$ in $P_{c}(M, \omega)$, we have

$$
\begin{aligned}
& \frac{d}{d t} J_{\omega}\left(\varphi_{t}\right)=-\frac{1}{V} \int_{M} \dot{\varphi}_{t}\left(\omega_{\varphi}^{n}-\omega^{n}\right), \\
& \frac{d}{d t} F_{\omega}^{0}\left(\varphi_{t}\right)=-\frac{1}{V} \int_{M} \dot{\varphi}_{t} \omega_{\varphi}^{n} .
\end{aligned}
$$

(2) $F_{\omega, \mu}(\varphi), F_{\omega}^{0}(\varphi)$ and $v_{\omega, \mu}(\varphi)$ satisfy the cocycle condition:

$$
\begin{aligned}
F_{\omega, \mu}(\varphi)+F_{\omega_{\varphi}, \mu}(\psi-\varphi) & =F_{\omega, \mu}(\psi), \\
F_{\omega}^{0}(\varphi)+F_{\omega_{\varphi}}^{0}(\psi-\varphi) & =F_{\omega}^{0}(\psi), \\
v_{\omega, \mu}(\varphi)+v_{\omega_{\varphi}, \mu}(\psi-\varphi) & =v_{\omega, \mu}(\psi) .
\end{aligned}
$$

In (2), the last two equations follow directly from differentiation. For $F_{\omega_{\varphi}, \mu}$, we need to choose a corresponding function $h_{\varphi}$ parallel to $h_{0}$ in (2-1). Whenever $\omega_{\varphi}$ is smooth or conic along $D$, we can write Ric $\omega_{\varphi}=\mu \omega_{\varphi}+\Omega_{\varphi}$ or Ric $\omega_{\varphi}=$ $\mu \omega_{\varphi}+\sum_{i=1}^{k} 2 \pi\left(1-\beta_{i}\right)\left[D_{i}\right]+\Omega_{\varphi}$, where $\Omega_{\varphi}$ is not necessarily nonnegative. Then all the arguments in the smooth case will apply.

From (1) we have a useful corollary from W. Ding [1988]. 
Corollary 3.5. For $0<t<1$, we have

$$
J_{\omega}(t \varphi) \leq t^{(n+1) / n} J_{\omega}(\varphi) .
$$

Proof. Consider the path $\{t \varphi\}_{0 \leq t \leq 1}$. Then we have

$$
\frac{d}{d t} J_{\omega}(t \varphi)=-\frac{1}{V} \int_{M} \varphi\left(\omega_{t \varphi}^{n}-\omega^{n}\right)=\frac{I_{\omega}(t \varphi)}{t} \geq \frac{n+1}{n} \frac{J_{\omega}(t \varphi)}{t} .
$$

Integrate this inequality, and then the corollary follows.

Now we discuss some relations among these functionals and their behaviors under different background metrics. First we have a lemma on the generalized energy $J_{\omega}$; see [Li and Sun 2014] for its proof.

Lemma 3.6. Suppose $\omega_{2}=\omega_{1}+\sqrt{-1} \partial \bar{\partial} \varphi$. Then for any $\varphi \in P_{c}\left(M, \omega_{1}\right) \cap$ $P_{c}\left(M, \omega_{2}\right)$, we have

$$
\left|J_{\omega_{1}}(\varphi)-J_{\omega_{2}}(\varphi)\right| \leq C\left(\omega_{1}, \omega_{2}\right)
$$

From this lemma and the cocycle property of $F_{\omega, \mu}(\varphi)$ and $v_{\omega, \mu}(\varphi)$, we observe that the properties of boundedness from below and properness are independent of the choice of metrics in the same Kähler class.

Next we want to know the relation between $F_{\omega, \mu}(\varphi)$ and $v_{\omega, \mu}(\varphi)$. We want to prove that these two properties of the two functionals are actually equivalent. These are similar to the proofs by Berman [2013] and Li and Sun [2014], and we use the proof in [Li 2012].

Lemma 3.7. (1) There exists a constant $C>0$ such that

$$
v_{\omega, \mu}(\varphi) \geq \mu F_{\omega, \mu}(\varphi)-C .
$$

(2) Suppose $\psi$ solves $\omega_{\psi}^{n}=e^{H_{0}-\mu \varphi}$ by the Calabi-Yau theorem. Then we have

$$
\mu F_{\omega, \mu}(\varphi)+\frac{1}{V} \int_{M} H_{0} \omega^{n} \geq v_{\omega, \mu}(\psi) .
$$

In particular, by (1) and (2) we know that $F_{\omega, \mu}$ being bounded from below is equivalent to $v_{\omega, \mu}$ being bounded from below.

(3) In the case that $v_{\omega, \mu}(\varphi) \geq C_{1} J_{\omega}(\varphi)-C_{2}$, where $C_{1}, C_{2}>0$, there exist constants $c, C^{\prime}>0$ such that

$$
F_{\omega, \mu}(\varphi) \geq c v_{\omega, \mu}(\varphi)-C^{\prime}
$$


Proof. (1) We modify the expression of the twisted Mabuchi functional in the definition:

$$
\begin{aligned}
v_{\omega, \mu}(\varphi)= & \frac{1}{V} \int_{M} \log \frac{\omega_{\varphi}^{n}}{\omega^{n}} \omega_{\varphi}^{n}+\frac{1}{V} \int_{M} H_{0}\left(\omega^{n}-\omega_{\varphi}^{n}\right)+\mu\left(F_{\omega}^{0}(\varphi)+\frac{1}{V} \int_{M} \varphi \omega_{\varphi}^{n}\right) \\
= & \mu F_{\omega, \mu}(\varphi)+\frac{1}{V} \int_{M} H_{0} \omega^{n}+\frac{1}{V} \int_{M} \log \frac{\omega_{\varphi}^{n}}{\omega^{n}} \omega_{\varphi}^{n} \\
& \quad-\frac{1}{V} \int_{M}\left(H_{0}-\mu \varphi\right) \omega_{\varphi}^{n}+\log \left(\frac{1}{V} \int_{M} e^{H_{0}-\mu \varphi} \omega^{n}\right) \\
= & \mu F_{\omega, \mu}(\varphi)+\frac{1}{V} \int_{M} H_{0} \omega^{n}+\log \left(\frac{1}{V} \int_{M} e^{H_{0}-\mu \varphi-\log \left(\omega_{\varphi}^{n} / \omega^{n}\right)} \omega_{\varphi}^{n}\right) \\
& \quad-\frac{1}{V} \int_{M}\left(H_{0}-\mu \varphi-\log \frac{\omega_{\varphi}^{n}}{\omega^{n}}\right) \omega_{\varphi}^{n} .
\end{aligned}
$$

Then (1) follows from the concavity of the logarithm.

(2) Still making use of the definition and the cocycle property, we have

$$
\begin{aligned}
v_{\omega, \mu}(\psi) & =\frac{1}{V} \int_{M} \log \frac{\omega_{\psi}^{n}}{\omega^{n}} \omega_{\psi}^{n}+\frac{1}{V} \int_{M} H_{0}\left(\omega^{n}-\omega_{\psi}^{n}\right)+\mu\left(F_{\omega}^{0}(\psi)+\frac{1}{V} \int_{M} \psi \omega_{\psi}^{n}\right) \\
& =\frac{1}{V} \int_{M}\left(H_{0}-\mu \varphi\right) \omega_{\psi}^{n}+\frac{1}{V} \int_{M} H_{0}\left(\omega^{n}-\omega_{\psi}^{n}\right)+\mu\left(F_{\omega}^{0}(\psi)+\frac{1}{V} \int_{M} \psi \omega_{\psi}^{n}\right) \\
& =\frac{1}{V} \int_{M} H_{0} \omega^{n}+\mu\left(F_{\omega}^{0}(\varphi)-F_{\omega_{\psi}}^{0}(\varphi-\psi)+\frac{1}{V} \int_{M}(\psi-\varphi) \omega_{\psi}^{n}\right) \\
& =\frac{1}{V} \int_{M} H_{0} \omega^{n}+\mu\left(F_{\omega, \mu}(\varphi)+\log \left(\frac{1}{V} \int_{M} e^{H_{0}-\mu \varphi} \omega^{n}\right)-J_{\omega_{\psi}}(\varphi-\psi)\right) .
\end{aligned}
$$

Then (2) follows from $e^{H_{0}-\mu \varphi} \omega^{n}=\omega_{\psi}^{n}$ and $J_{\omega_{\psi}}(\varphi-\psi) \geq 0$.

(3) From the assumption, we have a small $\delta>0$ such that $v_{\omega, \mu+\delta}(\varphi)=v_{\omega, \mu}(\varphi)-$ $\delta(I-J)_{\omega}(\varphi)$ is bounded from below, and so is $F_{\omega, \mu+\delta}(\varphi)$ by (2). Then

$$
\begin{aligned}
F_{\omega, \mu}(\varphi) & =F_{\omega}^{0}(\varphi)-\frac{\mu+\delta}{\mu} \frac{1}{v+\delta} \log \left(\frac{1}{V} \int_{M} e^{H_{0}-(\mu+\delta) \frac{\mu}{\mu+\delta} \varphi} \omega^{n}\right) \\
& =F_{\omega}^{0}(\varphi)+\frac{\mu+\delta}{\mu}\left(F_{\omega, \mu+\delta}\left(\frac{\mu}{\mu+\delta} \varphi\right)-F_{\omega}^{0}\left(\frac{\mu}{\mu+\delta} \varphi\right)\right) \\
& \geq J_{\omega}(\varphi)-\frac{\mu+\delta}{\mu} J_{\omega}\left(\frac{\mu}{\mu+\delta} \varphi\right)-C^{\prime} \\
& \geq\left(1-\left(\frac{\mu}{\mu+\delta}\right)^{\frac{1}{n}}\right) J_{\omega}(\varphi)-C^{\prime},
\end{aligned}
$$

where the last inequality follows from Corollary 3.5. 
To prove the properness of the functionals in the case of the existence of the conic metric $\omega=\omega_{\varphi}$, we need to verify that they are bounded from above.

Theorem 3.8. If the singular Monge-Ampère equation (2-2) has a solution $\varphi$, i.e., there exists a conic Kähler metric $\omega_{\varphi}=\omega_{0}+\sqrt{-1} \partial \bar{\partial} \varphi$ satisfying (1-1), then $\varphi$ attains the minimum of the functional $F_{\omega_{0}, \mu}$ on the space $P_{c}\left(M, \omega_{0}\right)$. In particular $F_{\omega_{0}, \mu}$ is bounded from above.

Proof. A parallel result is proved in [Li and Sun 2014], but we'd like to extend Ding and Tian's proof [Ding and Tian 1992; Tian 2000] to our conic case. Let's consider the continuity path of the complex Monge-Ampère equation

$$
\left(\omega_{0}+\sqrt{-1} \partial \bar{\partial} \varphi_{t}\right)^{n}=e^{H_{0}-t \varphi_{t}} \omega_{0}^{n} .
$$

We know that when $t=\mu$ this equation is solvable. By [Brendle 2013], we know that it is also solvable when $t=0$. When $0<t<\mu$, by the implicit function theorem, we need to consider whether the linearized operator of (3-2), $\Delta_{t}+t$, is invertible. We know that in the smooth case, by Bochner's formula, as Ric $\omega_{t}>t \omega_{t}$, it is invertible and we can prove the openness of the solvable set for $t$. However, in the conic case, [Jeffres et al. 2016] gives a parallel result. By their argument, we have $\Delta_{t}$ as the Friedrichs extension of the Laplacian associated to $\omega_{t}=\omega_{0}+\sqrt{-1} \partial \bar{\partial} \varphi_{t}$ and $\lambda_{1}\left(-\Delta_{t}\right)>t$, so the openness is true. We can set $\left\{\varphi_{t}\right\}$ as a continuous family of solutions of (3-2), and then we can do computations as [Tian 2000] in a weak sense.

First, taking the derivative of (3-2) with respect to $t$, we have

$$
\Delta_{t} \dot{\varphi}_{t}=-\varphi_{t}-t \dot{\varphi_{t}},
$$

where $\Delta_{t}$ is in a weak sense as in [Jeffres et al. 2016]. As for all $t$, we have $\int_{M} e^{H_{0}-t \varphi_{t}} \omega_{0}^{n}=V$, and taking the derivative with respect to $t$ we get

$$
\int_{M}\left(\varphi_{t}+t \dot{\varphi}_{t}\right) e^{H_{0}-t \varphi_{t}} \omega_{0}^{n}=0 .
$$

Making use of the formulas in the beginning of this section, we have

$$
\begin{aligned}
\frac{d}{d t}\left(I_{\omega_{0}}\left(\varphi_{t}\right)-J_{\omega_{0}}\left(\varphi_{t}\right)\right) & \\
= & \frac{1}{V} \int_{M} \dot{\varphi}_{t}\left(\omega_{0}^{n}-\omega_{t}^{n}\right)-\frac{1}{V} \int_{M} \varphi_{t} \Delta_{t} \dot{\varphi}_{t} \omega_{t}^{n}-\frac{1}{V} \int_{M} \dot{\varphi}_{t}\left(\omega_{0}^{n}-\omega_{t}^{n}\right) \\
& =\frac{1}{V} \int_{M} \varphi_{t}\left(\varphi_{t}+t \dot{\varphi}_{t}\right) \omega_{t}^{n} \\
& =-\frac{d}{d t}\left(\frac{1}{V} \int_{M} \varphi_{t} e^{H_{0}-t \varphi_{t}} \omega_{0}^{n}\right)+\frac{1}{V} \int_{M} \dot{\varphi}_{t} e^{H_{0}-t \varphi_{t}} \omega_{0}^{n} \\
& =-\frac{d}{d t}\left(\frac{1}{V} \int_{M} \varphi_{t} \omega_{t}^{n}\right)-\frac{1}{t V} \int_{M} \varphi_{t} \omega_{t}^{n} .
\end{aligned}
$$


From this, we have

$$
\frac{d}{d t}\left(t\left(I_{\omega_{0}}\left(\varphi_{t}\right)-J_{\omega_{0}}\left(\varphi_{t}\right)\right)\right)-\left(I_{\omega_{0}}\left(\varphi_{t}\right)-J_{\omega_{0}}\left(\varphi_{t}\right)\right)=-\frac{d}{d t}\left(\frac{1}{V} \int_{M} \varphi_{t} \omega_{t}^{n}\right)
$$

and integrating this from 0 to $t$, we have

$$
t\left(I_{\omega_{0}}\left(\varphi_{t}\right)-J_{\omega_{0}}\left(\varphi_{t}\right)\right)-\int_{0}^{t}\left(I_{\omega_{0}}\left(\varphi_{s}\right)-J_{\omega_{0}}\left(\varphi_{s}\right)\right) d s=-\frac{t}{V} \int_{M} \varphi_{t} \omega_{t}^{n}
$$

By the definition, it's just

$$
-\int_{0}^{t}\left(I_{\omega_{0}}\left(\varphi_{s}\right)-J_{\omega_{0}}\left(\varphi_{s}\right)\right) d s=t\left(J_{\omega_{0}}\left(\varphi_{t}\right)-\frac{1}{V} \int_{M} \varphi_{t} \omega_{0}^{n}\right)=t F_{\omega_{0}}^{0}\left(\varphi_{t}\right)
$$

As we have $\int_{M} e^{H_{0}-\mu \varphi} \omega_{0}^{n}=V$, we can derive that $F_{\omega_{0}, \mu}(\varphi) \leq 0$.

Now we choose $\varphi$ such that $\omega_{\varphi}=\omega_{0}+\sqrt{-1} \partial \bar{\partial} \varphi$ is a smooth Kähler metric. Then we have

$$
\operatorname{Ric} \omega_{\varphi}=\mu \omega_{\varphi}+\Omega_{\varphi},
$$

where $\Omega_{\varphi}$ is not necessarily nonnegative. Comparing it with (1-1), we have

$$
\left(\omega_{\varphi}+\sqrt{-1} \partial \bar{\partial}(\varphi-\varphi)\right)^{n}=e^{h_{\varphi}-\mu(\varphi-\varphi)-\sum_{i=1}^{m}\left(1-\beta_{i}\right) \log \left\|S_{i}\right\|_{i}^{2}+c_{\varphi}} \omega_{\varphi}^{n},
$$

where we take

$$
\sqrt{-1} \partial \bar{\partial} h_{\varphi}=\Omega_{\varphi}-\Omega-\sum_{i=1}^{m}\left(1-\beta_{i}\right)\left(R\left(\|\cdot\|_{i}\right) .\right.
$$

Then all the arguments are parallel and we have $F_{\omega_{\varphi}, \mu}(\varphi-\varphi) \leq 0$. Now let's consider the case when $\omega_{\varphi}$ is conic along $D$. Here we have the equation

$$
\operatorname{Ric} \omega_{\varphi}=\mu \omega_{\varphi}+\sum_{i=1}^{k} 2 \pi\left(1-\beta_{i}\right)\left[D_{i}\right]+\Omega_{\varphi} .
$$

Comparing it with (1-1), we have

$$
\left(\omega_{\varphi}+\sqrt{-1} \partial \bar{\partial}(\varphi-\varphi)\right)^{n}=e^{h_{\varphi}-\mu(\varphi-\varphi)+c_{\varphi}} \omega_{\varphi}^{n},
$$

where we have $\sqrt{-1} \partial \bar{\partial} h_{\varphi}=\Omega_{\varphi}-\Omega$. In this case, all the arguments are similar to those in the smooth case and we get the same conclusion. Now by the cocycle condition, we have

$$
F_{\omega_{0}, \mu}(\varphi)=F_{\omega_{0}, \mu}(\varphi)-F_{\omega_{\varphi}, \mu}(\varphi-\varphi) \geq F_{\omega_{0}, \mu}(\varphi) .
$$




\section{4. $\log \alpha$-invariant and properness of twisted energies}

We want to prove the properness of the twisted Ding energy. First we introduce the $\log \alpha$-invariant, and then see how to use this invariant to prove the properness of the twisted Mabuchi energy in the case that $\mu$ is small. Then we make use of concavity of energies to prove the properness of energies in the general case.

Recall that the $\alpha$-invariant in the smooth case was introduced by Tian [1987]. In [Berman 2013; Jeffres et al. 2016] this invariant is generalized to conic case. We introduce the so-called $\log \alpha$-invariant here, following [Li and Sun 2014].

Definition 4.1. Fix a smooth volume form vol. For any Kähler class $[\omega]$, we define the $\log \alpha$-invariant by

$$
\begin{aligned}
& \alpha(\omega, D)=\sup \left\{\alpha>0: \exists C_{\alpha}<\infty\right. \text { such that } \\
& \left.\frac{1}{V} \int_{M} e^{\alpha(\sup \varphi-\varphi)} \frac{\text { vol }}{\prod_{i=1}^{m}\left|S_{i}\right|^{2\left(1-\beta_{i}\right)}} \leq C_{\alpha} \text { for any } \varphi \in P_{c}(M, \omega)\right\} .
\end{aligned}
$$

Berman [2013] has an estimate for the positive lower bound of the $\log \alpha$-invariant in the conic case; i.e., there exists a positive number $\alpha_{0}$ such that $\alpha(\omega, D) \geq \alpha_{0}>0$. Using this estimate, we can prove that the twisted Mabuchi energy is proper when $\mu$ is small enough.

\section{Theorem 4.2. Suppose}

$$
\alpha(\omega, D) \geq \alpha_{0}>\frac{n}{n+1} \mu>0 .
$$

Then we have

$$
v_{\omega_{0}, \mu}(\varphi) \geq \epsilon J_{\omega_{0}}(\varphi)-C,
$$

where $\epsilon, C$ are constants depending on $\alpha_{0}, \mu$.

Proof. Following [Jeffres et al. 2016; Li and Sun 2014; Tian 2000] and making use of the logarithm property, for $\frac{n}{n+1} \mu<\alpha<\alpha_{0}$ we have

$$
\begin{aligned}
\log C_{\alpha} & \geq \log \left(\frac{1}{V} \int_{M} e^{\alpha(\sup \varphi-\varphi)} \frac{e^{H_{0}} \omega_{0}^{n}}{\prod_{i=1}^{m}\left|S_{i}\right|^{2\left(1-\beta_{i}\right)}}\right) \\
& \geq \log \left(\frac{1}{V} \int_{M} e^{\alpha(\sup \varphi-\varphi)-\log \left(\left(\prod_{i=1}^{m}\left|S_{i}\right|^{2\left(1-\beta_{i}\right)} \omega_{\varphi}^{n}\right) / \omega_{0}^{n}\right)+H_{0}} \omega_{\varphi}^{n}\right) \\
& \geq \frac{1}{V} \int_{M}\left(H_{0}-\frac{\prod_{i=1}^{m}\left|S_{i}\right|^{2\left(1-\beta_{i}\right)} \omega_{\varphi}^{n}}{\omega_{0}^{n}}\right) \omega_{\varphi}^{n}+\frac{\alpha}{V} \int_{M}(\sup \varphi-\varphi) \omega_{\varphi}^{n} \\
& \geq \frac{1}{V} \int_{M}\left(H_{0}-\frac{\prod_{i=1}^{m}\left|S_{i}\right|^{2\left(1-\beta_{i}\right)} \omega_{\varphi}^{n}}{\omega_{0}^{n}}\right) \omega_{\varphi}^{n}+\alpha I_{\omega_{0}}(\varphi) .
\end{aligned}
$$


By the definition of twisted Mabuchi energy, we have

$$
\begin{aligned}
v_{\omega_{0}, \mu}(\varphi) & =\frac{1}{V} \int_{M} \log \frac{\omega_{\varphi}^{n}}{\omega_{0}^{n}} \omega_{\varphi}^{n}+\frac{1}{V} \int_{M} H_{0}\left(\omega_{0}^{n}-\omega_{\varphi}^{n}\right)-\mu\left(I_{\omega_{0}}(\varphi)-J_{\omega_{0}}(\varphi)\right) \\
& \geq \log C_{\alpha}+\frac{1}{V} \int_{M} H_{0} \omega_{0}^{n}+\alpha I_{\omega_{0}}(\varphi)-\mu\left(I_{\omega_{0}}(\varphi)-J_{\omega_{0}}(\varphi)\right) \\
& \geq\left(\alpha-\frac{n}{n+1} \mu\right) I_{\omega_{0}}(\varphi)-C \\
& \geq\left(\frac{n+1}{n} \alpha-\mu\right) J_{\omega_{0}}(\varphi)-C .
\end{aligned}
$$

Given the equivalence of the properness of the twisted Ding energy and the Mabuchi energy, we have an easy corollary.

Corollary 4.3. When $\alpha(\omega, D) \geq \alpha_{0}>\frac{n}{n+1} \mu>0$, we have

$$
F_{\omega_{0}, \mu}(\varphi) \geq \epsilon J_{\omega_{0}}(\varphi)-C,
$$

where $\epsilon, C$ are constants depending on $\alpha_{0}, \mu$.

Until now we only had the properness when $\mu$ is small enough. For the general case, we need to apply the continuity method and the concavity property of the energy which is shown below to increase $\mu$. Here is a lemma which allows us to increase $\mu$; see also [Li and Sun 2014].

Lemma 4.4. Suppose $0<\mu_{0}<\mu_{1}$, and write $\mu=(1-t) \mu_{0}+t \mu_{1}$, where $0 \leq t \leq 1$. We have

$$
\mu F_{\omega_{0}, \mu}(\varphi) \geq(1-t) \mu_{0} F_{\omega_{0}, \mu}(\varphi)+t \mu_{1} F_{\omega_{0}, \mu}(\varphi) .
$$

Proof. The inequality follows from the convexity of exponential functions.

Now we can prove our main theorem in this section; similar results also appear in [Li and Sun 2014; Tian 2015].

Theorem 4.5. For $t \in(0, \mu]$ and any $\varphi \in P_{c}\left(M, \omega_{0}\right)$ there exist constants $\epsilon, C_{\epsilon}$ such that

$$
F_{\omega_{0}, t}(\varphi) \geq \epsilon J_{\omega_{0}}(\varphi)-C_{\epsilon} .
$$

Proof. We apply the continuity path similar to [Jeffres et al. 2016], i.e., (3-2). In our case, we may assume that $\Omega \neq 0$. Then by that paper, we have that $\lambda_{1}\left(-\Delta_{t}\right)>t$ for all $t \in(0, \mu]$, which allows us to prove the openness at $t=\mu$. So now when $\bar{\mu}=\mu+\delta$, where $\delta$ is very small, we have a solution $\bar{\varphi}$ for (3-2), where $\mu$ is replaced by $\bar{\mu}$. By Theorem 3.8, $F_{\omega_{0}, \bar{\mu}}(\varphi)$ is bounded from below. Since we have the corollary above, which asserts that when $t>0$ is very small $F_{\omega_{0}, t}(\varphi)$ is proper, 
by the lemma above, we know that for all $t \in(0, \mu]$ the twisted Ding energy is proper, i.e.,

$$
F_{\omega_{0}, t}(\varphi) \geq \epsilon J_{\omega_{0}}(\varphi)-C_{\epsilon}
$$

\section{5. $C^{\mathbf{0}}$-estimate for approximating solution: the case $\mu>0$}

Recall that in Section 2 we set up the approximating complex Monge-Ampère equation (2-3), which is expected to give us a smooth approximation of the conic Kähler metric $\omega=\omega_{0}+\sqrt{-1} \partial \bar{\partial} \varphi$. We also proved a $C^{0}$-estimate for $\varphi_{\delta}$ when $\mu \leq 0$. In this section, we want to make use of the properness of corresponding Lagrangians to prove the $C^{0}$-estimate when $\mu>0$. The first step is to prove the properness of the new approximating twisted Ding energy, which can be deduced from Section 4.

Lemma 5.1. For $t \in(0, \mu]$ we introduce the new approximating twisted Ding energy

$$
F_{\delta, t}(\varphi)=J_{\omega_{0}}(\varphi)-\frac{1}{V} \int_{M} \varphi \omega_{0}^{n}-\frac{1}{t} \log \left(\frac{1}{V} \int_{M} e^{h_{\delta}-t \varphi} \omega_{0}^{n}\right),
$$

which is the Lagrangian of the approximating complex Monge-Ampère equation (2-4) in the continuity path. Then we have

$$
F_{\delta, t}(\varphi) \geq \epsilon J_{\omega_{0}}(\varphi)-C(\epsilon, \delta, t) .
$$

Proof. At the end of Section 4, we proved that

$$
F_{\omega_{0}, t}(\varphi) \geq \epsilon J_{\omega_{0}}(\varphi)-C_{\epsilon} .
$$

Note that

$$
\begin{aligned}
h_{\delta} & =h_{0}-\sum_{i=1}^{m}\left(1-\beta_{i}\right) \log \left(\delta+\left\|S_{i}\right\|_{i}^{2}\right)+c_{\delta} \\
& \leq h_{0}-\sum_{i=1}^{m}\left(1-\beta_{i}\right) \log \left\|S_{i}\right\|_{i}^{2}+c_{\delta} \\
& =H_{0}-c+c_{\delta} .
\end{aligned}
$$

We have

$$
F_{\delta, t}(\varphi) \geq F_{\omega_{0}, t}(\varphi)+\frac{c-c_{\delta}}{t}
$$

and the lemma follows very easily.

Now we will follow [Tian 2000] to finish the $C^{0}$-estimate for $\varphi_{\delta}$. Similar to (3-4), we have

$$
-\int_{0}^{t}\left(I_{\omega_{0}}\left(\varphi_{\delta, s}\right)-J_{\omega_{0}}\left(\varphi_{\delta, s}\right)\right) d s=t\left(J_{\omega_{0}}\left(\varphi_{\delta, t}\right)-\frac{1}{V} \int_{M} \varphi_{\delta, t} \omega_{0}^{n}\right)=t F_{\omega_{0}}^{0}\left(\varphi_{\delta, t}\right),
$$


where $\varphi_{\delta, t}$ solves (2-4). By this equation, we can estimate

$$
\begin{aligned}
F_{\delta, \mu}\left(\varphi_{\delta, t}\right) & =F_{\omega_{0}}^{0}\left(\varphi_{\delta, t}\right)-\log \left(\frac{1}{V} \int_{M} e^{h_{\delta}-\mu \varphi_{\delta, t}} \omega_{0}^{n}\right) \\
& \leq-\log \left(\frac{1}{V} \int_{M} e^{h_{\delta}-t \varphi_{\delta, t}-(\mu-t) \varphi_{\delta, t}} \omega_{0}^{n}\right) \\
& =-\log \left(\frac{1}{V} \int_{M} e^{-(\mu-t) \varphi_{\delta, t}} \omega_{\delta, t}^{n}\right) \\
& \leq \frac{\mu-t}{\mu} \frac{1}{V} \int_{M} \varphi_{\delta, t} \omega_{\delta, t}^{n} .
\end{aligned}
$$

To finish the estimate, we need a useful lemma.

Lemma 5.2. $\left\|\varphi_{\delta, t}\right\|_{C^{0}} \leq C\left(1+J_{\omega_{0}}\left(\varphi_{\delta, t}\right)\right)$.

Proof. First we note that Ric $\omega_{\delta, t}>t$, and the volume is preserved. Then we have uniform Sobolev and Poincaré constants when $t$ doesn't tend to 0 . We observe that $n+\Delta_{0} \varphi_{\delta, t}>0$; then we get

$$
0 \leq \sup \varphi_{\delta, t} \leq \frac{1}{V} \int_{M} \varphi_{\delta, t} \omega_{0}^{n}+C
$$

by Green's formula. On the other hand, we have $n-\Delta_{\delta, t} \varphi_{\delta, t}>0$; by Moser's iteration, we have

$$
-\inf \varphi_{\delta, t} \leq-\frac{C}{V} \int_{M} \varphi_{\delta, t} \omega_{\delta, t}^{n}+C .
$$

By the normalization condition, when $\varphi_{\delta, t}$ changes sign, we have

$$
\left\|\varphi_{\delta, t}\right\|_{C^{0}} \leq \sup \varphi_{\delta, t}-\inf \varphi_{\delta, t} \leq C\left(1+I_{\omega_{0}}\left(\varphi_{\delta, t}\right)\right) \leq C\left(1+J_{\omega_{0}}\left(\varphi_{\delta, t}\right)\right) .
$$

In the proof we have

$$
0 \leq-\inf \varphi_{\delta, t} \leq-\frac{C}{V} \int_{M} \varphi_{\delta, t} \omega_{\delta, t}^{n}+C
$$

then we have

$$
\frac{1}{V} \int_{M} \varphi_{\delta, t} \omega_{\delta, t}^{n} \leq C
$$

which gives $F_{\delta, \mu}\left(\varphi_{\delta, t}\right) \leq C$. Combining the two lemmas above, we have the $C^{0}$-estimate for $\varphi_{\delta}$ and get the following result.

Theorem 5.3. For each $\delta>0$, the approximating complex Monge-Ampère equation (2-3) has a unique smooth solution $\varphi_{\delta}$, which gives us a smooth Kähler metric $\omega_{\delta}=\omega_{0}+\sqrt{-1} \partial \bar{\partial} \varphi_{\delta}$ such that $\operatorname{Ric} \omega_{\delta} \geq \mu \omega_{\delta}$. 


\section{Convergence when $\delta$ tends to 0}

In Section 5 we proved a $C^{0}$-estimate for $\varphi_{\delta}$. We also noted that in the approximating complex Monge-Ampère equation (2-3), the constant $c_{\delta}$ is uniformly bounded. Then the constant $C(\epsilon, \delta, t)$ in Lemma 5.1 is uniform with respect to $\delta$. According to this observation, we conclude that our $C^{0}$-estimate for $\varphi_{\delta}$ is uniform with respect to $\delta$, i.e., $\sup \left|\varphi_{\delta}\right| \leq C_{0}$. Based on this, we can give a $C^{2}$-estimate for $\varphi_{\delta}$ by the generalized Schwarz lemma first.

Lemma 6.1. We have

$$
C_{1} \omega_{0} \leq \omega_{\delta} \leq \frac{C_{2} \omega_{0}}{\prod_{i=1}^{m}\left(\delta+\left\|S_{i}\right\|^{2}\right)^{\left(1-\beta_{i}\right)}} .
$$

Proof. First we have $\sup \left|\varphi_{\delta}\right| \leq C_{0}$ and Ric $\omega_{\delta} \geq \mu \omega_{\delta}$. Take $\Delta$ as the Laplacian for $\omega_{\delta}$ and take a normal coordinate around a point $p$ for $\omega_{\delta}$, i.e., $g_{i \bar{j}}(p)=\delta_{i j}$, $d g_{i \bar{j}}(p)=0$. We may also take $g_{0 i \bar{j}}(p)=g_{0 i \bar{\imath}} \delta_{i j}$, i.e., diagonal for $\omega_{0}$. Then

$$
\begin{aligned}
\Delta \operatorname{tr}_{\omega_{\delta}} \omega_{0} & =g^{i \bar{l}}\left(g^{k \bar{l}} g_{0 k \bar{l}}\right)_{i \bar{l}} \\
& =g^{i \bar{l}}\left(g^{k \bar{k}}\right)_{i \bar{l}} g_{0 k \bar{k}}+g^{i \bar{l}} g^{k \bar{k}}\left(g_{0 k \bar{k}}\right)_{i \bar{l}} \\
& =g^{i \bar{l}} R_{i \bar{l}}^{k \bar{k}}(g) g_{0 k \bar{k}}-g^{i \bar{l}} g^{k \bar{k}} R_{i \bar{l} k \bar{k}}\left(g_{0}\right)+g^{i \bar{l}} g^{k \bar{k}} g^{l \bar{l}}\left(g_{0 k \bar{l}}\right)_{i}\left(g_{0 l \bar{k}}\right)_{\bar{l}} \\
& =R^{k \bar{k}} g_{0 k \bar{k}}-g^{i \bar{l}} g^{k \bar{k}} R_{i \bar{l} k \bar{k}}\left(g_{0}\right)+g_{0}^{i \bar{l}} g^{k \bar{k}} g^{l \bar{l}}\left(g_{0 k \bar{l}}\right)_{i}\left(g_{0 l \bar{k}}\right)_{\bar{l}} \\
& \geq-g^{i \bar{l}} g^{k \bar{k}} R_{i \bar{l} k \bar{k}}\left(g_{0}\right)+g_{0}^{i \bar{l}} g^{k \bar{k}} g^{l \bar{l}}\left(g_{0 k \bar{l}}\right)_{i}\left(g_{0 l \bar{k}}\right)_{\bar{l}},
\end{aligned}
$$

and the last inequality follows from Ric $\omega_{\delta} \geq \mu \omega_{\delta}$. Now we have

$$
\begin{aligned}
\Delta \log \operatorname{tr}_{\omega_{\delta}} \omega_{0} & =\frac{\Delta \operatorname{tr}_{\omega_{\delta}} \omega_{0}}{\operatorname{tr}_{\omega_{\delta}} \omega_{0}}-\frac{\left|\nabla \operatorname{tr}_{\omega_{\delta}} \omega_{0}\right|^{2}}{\left|\operatorname{tr}_{\omega_{\delta}} \omega_{0}\right|^{2}} \\
& \geq \frac{\left(\operatorname{tr}_{\omega_{\delta}} \omega_{0}\right) g_{0}^{i \bar{l}} g^{k \bar{k}} g^{l \bar{l}}\left(g_{0 k \bar{l}}\right)_{i}\left(g_{0 l \bar{k}}\right)_{\bar{l}}-g^{i \bar{\imath}} g^{k \bar{k}} g^{l \bar{l}}\left(g_{0 k \bar{k}}\right)_{i}\left(g_{0 l \bar{l}}\right)_{\bar{l}}}{\left|\operatorname{tr}_{\omega_{\delta}} \omega_{0}\right|^{2}} \\
& -\frac{g^{i \bar{l}} g^{k \bar{k}} R_{i \bar{l} k \bar{k}}\left(g_{0}\right)}{\operatorname{tr}_{\omega_{\delta}} \omega_{0}} \\
& \geq-a \operatorname{tr}_{\omega_{\delta}} \omega_{0},
\end{aligned}
$$

where the bisectional curvature of $\omega_{0}$ is less than $a$ and the last inequality follows from $g_{0}^{i \bar{l}} \operatorname{tr}_{\omega_{\delta}} \omega_{0} \geq g^{i \bar{l}}$. As we have sup $\left|\varphi_{\delta}\right| \leq C_{0}$, we take $u=\log \operatorname{tr}_{\omega_{\delta}} \omega_{0}-(a+1) \varphi_{\delta}$. Then we will have

$$
\Delta u \geq \operatorname{tr}_{\omega_{\delta}} \omega_{0}-n(a+1)=e^{u+n(a+1)}-n(a+1) .
$$


By the maximal principle $u \leq C(a)$, and we then get $\operatorname{tr}_{\omega_{\delta}} \omega_{0} \leq C^{\prime}$, which will give us that $C_{1} \omega_{0} \leq \omega_{\delta}$. For the other side, making use of the complex Monge-Ampère equation (2-3) and the inequality we obtained, we can easily deduce that

$$
\omega_{\delta} \leq \frac{C_{2} \omega_{0}}{\prod_{i=1}^{m}\left(\delta+\left\|S_{i}\right\|^{2}\right)^{\left(1-\beta_{i}\right)}} .
$$

From this lemma, by the $C^{3}$-estimate in [Yau 1978] (or see [Tian 2000]) and regularity theory we can prove that for any $l>2$ and compact set $K \in M \backslash D$, there exists a uniform constant $C(l, K)$ such that we have a high order estimate locally:

$$
\left\|\varphi_{\delta}\right\| \leq C(l, K) .
$$

As we have all the estimates we need, we can prove the main theorem below, following [Tian 2015].

Theorem 6.2. As $\delta$ tends to 0 , the smooth Kähler metric $\omega_{\delta}$ converges to the conic Kähler metric $\omega$ in the Gromov-Hausdorff topology on $M$ and in the smooth topology outside the divisor D.

Proof. We first consider the case that $D$ is an irreducible divisor. As we have high order estimates (6-1) and (6-2) outside the divisor $D$, it suffices to prove $\omega_{\delta}$ converges to $\omega$ in the Gromov-Hausdorff topology. For all $\omega_{\delta}$ we have Ric $\omega_{\delta} \geq \mu$, $\operatorname{Vol}\left(M, \omega_{\delta}\right)=V$; to apply the compactness theorem of Cheeger-Gromov (e.g., see Chapter 10 in [Petersen 2006]), we only need to bound the diameter for all $\omega_{\delta}$. In the case that $\mu>0$ we can get it directly by Meyer's theorem. However, as we have the estimate (6-1), it's easy to control the length of arbitrary geodesics outside the divisor. And in the neighborhood of some irreducible divisor, say $D$, we make use of local coordinates and set $r=\left|z_{1}\right|$, where $\left\{z_{1}=0\right\}$ locally defines the divisor $D$. Now we know that $\|S\|$ here is almost $r$ near the divisor and we consider the length of a short geodesic $\gamma$ transverse to $D$ such that

$$
L\left(\gamma, \omega_{\delta}\right) \approx C \int_{0}^{r_{0}} \frac{d r}{\left(\delta+r^{2}\right)^{\frac{1-\beta}{2}}} \leq C \int_{0}^{r_{0}} \frac{d r}{r^{1-\beta}} \leq \frac{C r_{0}^{\beta}}{\beta} .
$$

Along the geodesics almost tangential to $D$ we almost have $d z_{1}=0$ so in all cases the diameter with respect to $\omega_{\delta}$ is uniformly bounded. Now by the compactness theorem, without loss of generality, $\left(M, \omega_{\delta}\right)$ converges to a length space $(\bar{M}, \bar{d})$ in the Gromov-Hausdorff topology. To prove the theorem we need to prove that $(\bar{M}, \bar{d})$ coincides with $(M, \omega)$. As we have high order estimate (6-2) outside the divisor $D$, there exists an open set $U$ in $\bar{M}$ which is equivalent to $M \backslash D$, and the equivalence $i: M \backslash D \rightarrow U$ induces an isometry between $M \backslash D,\left.\omega\right|_{M \backslash D}$ and $(U, \bar{d})$. Now we note that $M \backslash D$ is geodesically convex with respect to $\omega$, i.e., given any two points $p, q \in M \backslash D$, there exists a minimal geodesic $\gamma \subset M \backslash D$ 
joining them. Actually we only need to consider the case when $p, q$ are in the small neighborhood of $o \in D$. In this case we know that the metric $\omega$ is almost the standard conic metric around a point $o \in D$, which behaves like

$$
\omega_{o, c}=\sqrt{-1}\left(\frac{d z_{1} \wedge d \bar{z}_{1}}{\left|z_{1}\right|^{2(1-\beta)}}+\sum_{i=2}^{n} d z_{i} \wedge d \bar{z}_{i}\right) .
$$

Now we assume that $\left|z_{1}(p)\right|=\left|z_{1}(q)\right|=\epsilon$ and $\left|z_{i}(p)\right|,\left|z_{i}(q)\right| \approx \epsilon$, where $\epsilon>0$ is small enough and $2 \geq i \geq n$. First we choose the segment connecting $p$ and $q$ across the point $o \in D$. By the estimate above we know that

$$
d(p, o)+d(o, q) \approx \frac{2 \epsilon^{\beta}}{\beta} .
$$

On the other hand we choose a segment $\gamma^{\prime}$ whose projection on the $z_{1}$ coordinate is almost a geodesic in the cone with angle $\beta$; by standard computation we know that

$$
L\left(\gamma^{\prime}\right) \approx C \epsilon+2 \sin \frac{\pi \beta}{2} \frac{\epsilon^{\beta}}{\beta} .
$$

As $\epsilon$ is small and $\beta<1$, we conclude that the geodesic connecting $p$ and $q$ doesn't cross the point $o \in D$. In the general case we only need to choose $p^{\prime}, q^{\prime}$ as in the case above to replace $p, q$ and connect $p, p^{\prime}$ and $q, q^{\prime}$ respectively. Then the rest of the argument follows.

As $M \backslash D$ is geodesically convex, by the $C^{2}$-estimate in (6-1), we can estimate as above to show that for each point $o \in D$, a radical short line connecting $o$ and a point outside the divisor is always rectifiable and absolutely continuous with respect to local coordinates; thus we can see that $M$ is the metric completion of $M \backslash D$. Moreover, the equivalence $i$ extends to a Lipschitz map from $(M, \omega)$ onto $(\bar{M}, \bar{d})$ (we still denote this map by $i$ ) and the Lipschitz constant is 1 . What remains to do is to prove $i$ is an isometry between $(M, \omega)$ and $(\bar{M}, \bar{d})$. As $(\bar{M}, \bar{d})$ is a metric completion of $M \backslash D$, we only need to prove that for $p, q \in M \backslash D$,

$$
d_{\omega}(p, q)=\bar{d}(i(p), i(q)) .
$$

Observe that $\bar{D}=i(D)$ is the Gromov-Hausdorff limit of $D$ under the convergence of $\left(M, \omega_{\delta}\right)$ to $(\bar{M}, \bar{d})$, whose Hausdorff measure is 0 , by the $C^{2}$-estimate in (6-1). Now we only need to prove that for any $\bar{p}, \bar{q} \in \bar{M} \backslash \bar{D}$ there exists a minimizing geodesic $\gamma \subset \bar{M} \backslash \bar{D}$ joining $\bar{p}, \bar{q}$. If not, we will have

$$
\bar{d}(\bar{p}, \bar{q})<d_{\omega}(p, q),
$$

where $\bar{p}=i(p), \bar{q}=i(q)$. Then there exists a small $r>0$ such that

(1) $B_{r}(\bar{p}, \bar{d}) \cap \bar{D}=\varnothing, B_{r}(\bar{q}, \bar{d}) \cap \bar{D}=\varnothing$, where $B_{r}(\cdot, \bar{d})$ is a geodesic ball in $(\bar{M}, \bar{d})$; 
(2) $\bar{d}(\bar{x}, \bar{y})<d_{\omega}(x, y)$, where $\bar{x}=i(x) \in B_{r}(\bar{p}, \bar{d})$ and $\bar{y}=i(y) \in B_{r}(\bar{q}, \bar{d})$.

From these two we know that any minimizing geodesic $\gamma$ connecting $\bar{x}$ and $\bar{y}$ intersects $\bar{D}$. As $r>0$ is small, and $i$ is an isometry outside the divisor $D$, we have

$$
B_{r}(\bar{p}, \bar{d})=i\left(B_{r}(p, \omega)\right), \quad B_{r}(\bar{q}, \bar{d})=i\left(B_{r}(q, \omega)\right) .
$$

Choose a small tubular neighborhood $T$ of $D$ in $M$ whose closure is disjoint from both $B_{r}(p, \omega)$ and $B_{r}(q, \omega)$. When the radius of such a tubular neighborhood is small enough we can make Vol $\partial T$ arbitrarily small. Now we can choose $p_{\delta}, q_{\delta} \in M$ and a neighborhood $T_{\delta}$ of $D$ with respect to $\omega_{\delta}$ such that as $\delta \rightarrow 0, p_{\delta}, q_{\delta}, T_{\delta}$ converge to $\bar{p}, \bar{q}, i(T)$ in the Gromov-Hausdorff topology. By the volume convergence theorem of Colding, $\lim _{\delta \rightarrow 0+} \operatorname{Vol}\left(\partial T_{\delta}, \omega_{\delta}\right)=\operatorname{Vol}(\partial T, \omega)$, so $\operatorname{Vol}\left(\partial T_{\delta}, \omega_{\delta}\right)$ can also be arbitrarily small as $\delta \rightarrow 0$. Also by convergence, when $\delta$ is small enough, $B_{r}\left(p_{\delta}, \omega_{\delta}\right), B_{r}\left(q_{\delta}, \omega_{\delta}\right)$ and $T_{\delta}$ are mutually disjoint. By (2), any minimizing geodesic $\gamma_{\delta}$ connecting any $w \in B_{r}\left(p_{\delta}, \omega_{\delta}\right)$ and $z \in B_{r}\left(q_{\delta}, \omega_{\delta}\right)$ intersects $T_{\delta}$. Now we need an estimate due to Gromov:

Lemma 6.3. We have

$$
c(\mu) r^{2 n} \leq \operatorname{Vol}\left(B_{r}\left(q_{\delta}, \omega_{\delta}\right), \omega_{\delta}\right) \leq C(L, \mu, n, r) \operatorname{Vol}\left(\partial T_{\delta}, \omega_{\delta}\right),
$$

where $L=\bar{d}(\bar{p}, \bar{q})$.

Proof. The first inequality follows from the Ricci lower bound and Gromov's relative volume comparison theorem directly. For the second inequality, by Chapter 9 in [Petersen 2006], we set $\lambda(t, \theta)$ as the volume density function, where $t$ is the distance from $p_{\delta}$. We also set $\lambda_{k}(t, \theta)$ as the standard volume density function of the space form with constant curvature $k=\mu /(n-1)$. By the argument in [Petersen 2006] we know that the map $t \rightarrow \lambda(t, \theta) / \lambda_{k}(t, \theta)$ is nonincreasing in $t$. In our case, we consider the geodesics from $p_{\delta}$ to $z \in B_{r}\left(q_{\delta}, \omega_{\delta}\right)$. According to the construction, we have $r<d\left(p_{\delta}, z_{T}\right)<d\left(p_{\delta}, z\right), L-r<d\left(p_{\delta}, z\right)<L+r$, where $z_{T}$ is the intersection point of the geodesics from $p_{\delta}$ to $z$ and $\partial T_{\delta}$, and $L \approx d\left(p_{\delta}, q_{\delta}\right)$. Along $\partial T_{\delta}$, we have

$$
\frac{\lambda\left(z_{T}\right)}{\lambda_{k}\left(z_{T}\right)} \geq \frac{\lambda(z)}{\lambda_{k}(z)} .
$$

Let $S \in S^{2 n-1}$, let $C(S)$ denote the part where all the geodesics from $p_{\delta}$ to $z \in B_{r}\left(q_{\delta}, \omega_{\delta}\right)$ lie in the corresponding geodesic cone, and let $t(\theta)$ be the distance from $p_{\delta}$ to each point of $\partial T_{\delta}$. Then we have

$$
\begin{aligned}
\operatorname{Vol} \partial T_{\delta} \geq \int_{\partial T_{\delta} \cap C(S)} \lambda(t, \theta) & =\int_{S} t^{2 n-1}(\theta) \lambda(t, \theta) d \theta \\
& \geq \int_{S} \lambda\left(L^{\prime}\right) \frac{\lambda_{k}(t(\theta))}{\lambda_{k}\left(L^{\prime}\right)} t^{2 n-1}(\theta) d \theta \geq C \int_{S} \lambda\left(L^{\prime}\right) L^{\prime 2 n-1} d \theta,
\end{aligned}
$$


where $L-r<L^{\prime}<L+r$. Taking the integral of this inequality yields $\operatorname{Vol} \partial T_{\delta} \geq \frac{2 C}{r} \int_{L-r}^{L+r} \int_{S} \lambda\left(L^{\prime}\right) L^{\prime 2 n-1} d \theta d t \geq C(L, \mu, n, r) \operatorname{Vol}\left(B_{r}\left(q_{\delta}, \omega_{\delta}\right), \omega_{\delta}\right)$.

Then the lemma follows.

Since we know that $\operatorname{Vol}\left(\partial T_{\delta}, \omega_{\delta}\right)$ can also be arbitrarily small as $\delta$ tends to 0 , the lemma above leads to a contradiction. Then $i$ can extend to an isometry from $(M, \omega)$ onto $(\bar{M}, \bar{d})$, and the theorem follows when $D$ is irreducible. In the case that $D$ is a simple normal crossing divisor, we observe that near the crossing point $o$, the model metric can be rewritten as

$$
\begin{aligned}
\omega_{o, c} & =\sqrt{-1}\left(\sum_{i=1}^{m} \frac{d z_{i} \wedge d \bar{z}_{i}}{\left|z_{i}\right|^{2\left(1-\beta_{i}\right)}}+\sum_{i=m+1}^{n} d z_{i} \wedge d \bar{z}_{i}\right) \\
& =\sqrt{-1}\left(\sum_{i=1}^{m} \frac{d z_{i}^{\beta_{i}} \wedge d \bar{z}_{i}^{\beta_{i}}}{\beta_{i}^{2}}+\sum_{i=m+1}^{n} d z_{i} \wedge d \bar{z}_{i}\right) .
\end{aligned}
$$

For $1 \leq i \leq m$, if we take $w_{i}:=z_{i}^{\beta_{i}} / \beta_{i}$, we can realize the original conic metric as a Euclidean metric under these new coordinates. To find the minimal geodesic we then only need to project two points in the original space to each coordinate direction; if in each direction we can find a minimal geodesic, we are done. In this case we deduce the problem to the one irreducible divisor case. Obviously, for the conic metric along a simple normal crossing divisor, the minimal geodesic will always lie in the regular part. Hence in the general case the theorem still follows.

\section{Acknowledgments}

The author thanks his Ph.D. thesis advisor, Professor Gang Tian, for a lot of discussions and encouragement, and Yanir Rubinstein and Chi Li for many useful conversations. He also thanks CSC for partial financial support during his Ph.D. career.

\section{References}

[Berman 2013] R. J. Berman, "A thermodynamical formalism for Monge-Ampère equations, MoserTrudinger inequalities and Kähler-Einstein metrics", Adv. Math. 248 (2013),1254-1297. MR 3107540 Zbl 1286.58010

[Brendle 2013] S. Brendle, "Ricci flat Kähler metrics with edge singularities", Int. Math. Res. Not. 2013:24 (2013), 5727-5766. MR 3144178 Zbl 1293.32029

[Cheeger et al. 2002] J. Cheeger, T. H. Colding, and G. Tian, "On the singularities of spaces with bounded Ricci curvature”, Geom. Funct. Anal. 12:5 (2002), 873-914. MR 1937830 Zbl 1030.53046

[Chen et al. 2015a] X. X. Chen, S. Donaldson, and S. Sun, "Kähler-Einstein metrics on Fano manifolds, I: Approximation of metrics with cone singularities", J. Amer. Math. Soc. 28:1 (2015), 183-197. MR 3264766 Zbl 1312.53096 
[Chen et al. 2015b] X. X. Chen, S. Donaldson, and S. Sun, "Kähler-Einstein metrics on Fano manifolds, II: Limits with cone angle less than 2 $\pi$ ", J. Amer. Math. Soc. 28:1 (2015), 199-234. MR 3264767 Zbl 1312.53097

[Chen et al. 2015c] X. X. Chen, S. Donaldson, and S. Sun, "Kähler-Einstein metrics on Fano manifolds, III: Limits as cone angle approaches $2 \pi$ and completion of the main proof", J. Amer. Math. Soc. 28:1 (2015), 235-278. MR 3264768 Zbl 1311.53059

[Ding 1988] W. Y. Ding, "Remarks on the existence problem of positive Kähler-Einstein metrics", Math. Ann. 282:3 (1988), 463-471. MR 967024 Zbl 0661.53045

[Ding and Tian 1992] W. Y. Ding and G. Tian, "Kähler-Einstein metrics and the generalized Futaki invariant”, Invent. Math. 110:2 (1992), 315-335. MR 1185586 Zbl 0779.53044

[Jeffres et al. 2016] T. Jeffres, R. Mazzeo, and Y. A. Rubinstein, "Kähler-Einstein metrics with edge singularities", Ann. of Math. (2) 183:1 (2016), 95-176. MR 3432582 Zbl 06541584

[Li 2012] C. Li, Kähler-Einstein metrics and K-stability, Ph.D. thesis, Princeton University, 2012, available at http://www.math.sunysb.edu/ chili/papers/PhDthesis.pdf.

[Li and Sun 2014] C. Li and S. Sun, "Conical Kähler-Einstein metrics revisited”, Comm. Math. Phys. 331:3 (2014), 927-973. MR 3248054 Zbl 1296.32008

[Petersen 2006] P. Petersen, Riemannian geometry, 2nd ed., Graduate Texts in Mathematics 171, Springer, New York, NY, 2006. MR 2243772 Zbl 1220.53002

[Tian 1987] G. Tian, "On Kähler-Einstein metrics on certain Kähler manifolds with $C_{1}(M)>0$ ", Invent. Math. 89:2 (1987), 225-246. MR 894378 Zbl 0599.53046

[Tian 2000] G. Tian, Canonical metrics in Kähler geometry, Birkhäuser, Basel, 2000. MR 1787650 Zbl 0978.53002

[Tian 2012] G. Tian, "Existence of Einstein metrics on Fano manifolds", pp. 119-159 in Metric and differential geometry, edited by X. Dai and X. Rong, Progress in Mathematics 297, Birkhäuser, Basel, 2012. MR 3220441 Zbl 1250.53044

[Tian 2015] G. Tian, "K-stability and Kähler-Einstein metrics”, Comm. Pure Appl. Math. 68:7 (2015), 1085-1156. MR 3352459 Zbl 1318.14038

[Yau 1978] S. T. Yau, "On the Ricci curvature of a compact Kähler manifold and the complex MongeAmpère equation, I”, Comm. Pure Appl. Math. 31:3 (1978), 339-411. MR 480350 Zbl 0369.53059

Received December 23, 2014. Revised September 24, 2015.

\section{LIANGMING SHEN}

DEPARTMENT OF MATHEMATiCS

PRINCETON UNIVERSITY

PRINCETON, NJ 08544

UNITED STATES

Current address:

Mathematical Sciences Research Institute

17 GAUSS WAY

BERKELEY, CA 94720

UNITED STATES

lmshen@math.ubc.ca 


\title{
PACIFIC JOURNAL OF MATHEMATICS
}

Founded in 1951 by E. F. Beckenbach (1906-1982) and F. Wolf (1904-1989)

$$
\text { msp.org/pjm }
$$

\section{EDITORS}

\author{
Don Blasius (Managing Editor) \\ Department of Mathematics \\ University of California \\ Los Angeles, CA 90095-1555 \\ blasius@math.ucla.edu
}

\author{
Paul Balmer \\ Department of Mathematics \\ University of California \\ Los Angeles, CA 90095-1555 \\ balmer@math.ucla.edu \\ Robert Finn \\ Department of Mathematics \\ Stanford University \\ Stanford, CA 94305-2125 \\ finn@math.stanford.edu \\ Sorin Popa \\ Department of Mathematics \\ University of California \\ Los Angeles, CA 90095-1555 \\ popa@math.ucla.edu
}

\author{
Vyjayanthi Chari \\ Department of Mathematics \\ University of California \\ Riverside, CA 92521-0135 \\ chari@math.ucr.edu \\ Kefeng Liu \\ Department of Mathematics \\ University of California \\ Los Angeles, CA 90095-1555 \\ liu@math.ucla.edu \\ Igor Pak \\ Department of Mathematics \\ University of California \\ Los Angeles, CA 90095-1555 \\ pak.pjm@gmail.com \\ Paul Yang \\ Department of Mathematics \\ Princeton University \\ Princeton NJ 08544-1000 \\ yang@math.princeton.edu
}

\section{PRODUCTION}

Silvio Levy, Scientific Editor, production@msp.org

\section{SUPPORTING INSTITUTIONS}

ACADEMIA SINICA, TAIPEI

CALIFORNIA INST. OF TECHNOLOGY

STANFORD UNIVERSITY

UNIV. OF BRITISH COLUMBIA

UNIV. OF CALIFORNIA, BERKELEY

UNIV. OF CALIFORNIA, DAVIS

UNIV. OF CALIFORNIA, LOS ANGELES

UNIV. OF CALIFORNIA, RIVERSIDE

UNIV. OF CALIFORNIA, SAN DIEGO

UNIV. OF CALIF., SANTA BARBARA
KEIO UNIVERSITY

MATH. SCIENCES RESEARCH INSTITUTE

NEW MEXICO STATE UNIV.

OREGON STATE UNIV.
Daryl Cooper

Department of Mathematics

University of California

Santa Barbara, CA 93106-3080 cooper@math.ucsb.edu

Jiang-Hua Lu

Department of Mathematics

The University of Hong Kong

Pokfulam Rd., Hong Kong

jhlu@maths.hku.hk

$$
\text { Jie Qing }
$$

Department of Mathematics

University of California

Santa Cruz, CA 95064

qing@ cats.ucsc.edu

\author{
UNIV. OF CALIF., SANTA CRUZ \\ UNIV. OF MONTANA \\ UNIV. OF OREGON \\ UNIV. OF SOUTHERN CALIFORNIA \\ UNIV. OF UTAH \\ UNIV. OF WASHINGTON \\ WASHINGTON STATE UNIVERSITY
}

These supporting institutions contribute to the cost of publication of this Journal, but they are not owners or publishers and have no responsibility for its contents or policies.

See inside back cover or msp.org/pjm for submission instructions.

The subscription price for 2016 is US $\$ 440 /$ year for the electronic version, and \$600/year for print and electronic.

Subscriptions, requests for back issues and changes of subscriber address should be sent to Pacific Journal of Mathematics, P.O. Box 4163, Berkeley, CA 94704-0163, U.S.A. The Pacific Journal of Mathematics is indexed by Mathematical Reviews, Zentralblatt MATH, PASCAL CNRS Index, Referativnyi Zhurnal, Current Mathematical Publications and Web of Knowledge (Science Citation Index).

The Pacific Journal of Mathematics (ISSN 0030-8730) at the University of California, c/o Department of Mathematics, 798 Evans Hall \#3840, Berkeley, CA 94720-3840, is published twelve times a year. Periodical rate postage paid at Berkeley, CA 94704, and additional mailing offices. POSTMASTER: send address changes to Pacific Journal of Mathematics, P.O. Box 4163, Berkeley, CA 94704-0163.

PJM peer review and production are managed by EditFLOW ${ }^{\circledR}$ from Mathematical Sciences Publishers.

PUBLISHED BY

\section{I. mathematical sciences publishers}

nonprofit scientific publishing

http://msp.org/

(C) 2016 Mathematical Sciences Publishers 


\section{PACIFIC JOURNAL OF MATHEMATICS}

Volume $284 \quad$ No. $2 \quad$ October 2016

Spherical CR Dehn surgeries

Miguel ACOsta

Degenerate flag varieties and Schubert varieties: a characteristic free approach

283

Giovanni Cerulli Irelli, Martina LANini and Peter

LITTELMANN

Solitons for the inverse mean curvature flow

Gregory Drugan, HoJoo LEE and GLEN WHEELER

Bergman theory of certain generalized Hartogs triangles

\section{LUKE D. EDHOLM}

Transference of certain maximal Hilbert transforms on the torus

DASHAN FAN, HUOXIONG WU and FAYOU ZHAO

The Turaev and Thurston norms

STEFAN FriedL, DANIEL S. Silver and Susan G. WiLLiams

A note on nonunital absorbing extensions

JAMES GABE

On nonradial singular solutions of supercritical biharmonic equations

Zongming GuO, JUNCHENG WeI and Wen YANG

Natural commuting of vanishing cycles and the Verdier dual

\section{DAVID B. MASSEY}

The nef cones of and minimal-degree curves in the Hilbert schemes of points on certain surfaces

ZHENBo QIN and YUPING TU

Smooth approximation of conic Kähler metric with lower Ricci curvature bound

\section{LIANGMING SHEN}

Maps from the enveloping algebra of the positive Witt algebra to regular algebras

Susan J. SierRa and Chelsea Walton 